\title{
Mise en scène d'objets de nature à Paris et Varsovie : les arbres remarquables de deux forêts périurbaines
}

\author{
Paul Arnould ${ }^{\mathrm{a}}$, Caroline Cieslak ${ }^{\mathrm{b}}$ \\ a Géographe et historien, École normale supérieure Lettres et Sciences humaines, 15 parvis René Descartes, BP 7000, \\ 69342 Lyon cedex 07, France \\ b Géographe, CNRS-ENS, laboratoire Biogéo, 4 bis rue Eugène Schueller, 93600 Aulnay-sous-Bois
}

\begin{abstract}
Plus que d'autres, certains arbres sont l'objet de soins et d'attention, suscitant un sentiment de responsabilité, voire de fierté de la part des gestionnaires et du public. C'est en particulier le cas des arbres remarquables dans les forêts périurbaines. On peut s'interroger sur le statut de ces sujets d'exception - et sur leur gestion : conduit-elle à sacrifier le naturel au profit du culturel? Un thème abordé ici par deux géographes, à partir d'une démarche comparative originale.
\end{abstract}

La Rédaction

\section{Mots-clés :}

nature ;

aménagement

forestier;

arbres remarquables ;

biogéographie;

étude comparée ;

France :

Pologne

\section{Keywords:}

Nature;

forest planning; remarkable trees; biogeography; comparative studies; France;

Poland
Résumé - Les arbres remarquables, objets naturels hors normes, par leur âge, leur taille, leur aspect, leur histoire, sont devenus des produits de consommation culturelle. Ils sont nommés, photographiés, cartographiés, soignés. En tant qu'individus exceptionnels ils parlent plus à l'imaginaire que le massif forestier, société d'arbres anonymes. Comparer le statut des arbres remarquables dans deux grandes forêts périurbaines de deux capitales nationales, Paris et Varsovie, au travers des territoires emblématiques que sont Fontainebleau et Kampinos permet de démêler l'imbrication subtile du naturel et du culturel dans la mise en scène des objets de nature. C'est aussi l'occasion de s'interroger sur la délicate gestion de ces sujets. Ils rompent avec les logiques productivistes, chères à certains forestiers, pour imposer des approches patrimoniales, esthétiques liées à l'irrésistible croissance des fonctions de loisirs des urbains en forêt. Cette «arborimania » ne va pas sans excès. Elle favorise l'acharnement thérapeutique et valorise des idées fixistes au détriment des nécessaires visions dynamiques.

\begin{abstract}
Presentation of natural objects in Paris and Warsaw: remarkable trees of two periurban woods. Remarkable trees are unusual natural objects, exceptional because of their size, their appearance or their history. They have become products of cultural consumption. They are given names, they are photographed, mapped but, above all, protected and looked after. As exceptional individuals, they appeal to man's imagination much more than does an ordinary forest area, which is only a society of anonymous trees. This article compares the status of special trees in two large urban woods: Fontainebleau and Kampinos which are located near the capital cities Paris and Warsaw. These exceptional territories with their large number of visitors - enable us to study the fine interweaving of nature and culture in the representation of natural objects. One key question concerns the management of special trees, these outstanding individuals. They do not fit to the usual principles of high productivity which only aim at producing large quantities of wood. They require different approaches: aesthetic, relating to cultural heritage or to landscape, which are linked to the dramatic growth in the needs of urban inhabitants - in our leisure society - in search of nature, which they hope to find in woods. This "tree-mania" is not without its excesses. It contributes to the fight for the prolongation of the life of older trees at all costs; it favours a "fixist" view of nature, to the detriment of a dynamic conception. A tree is born, it lives and (usually) it dies. It is not eternal.
\end{abstract}



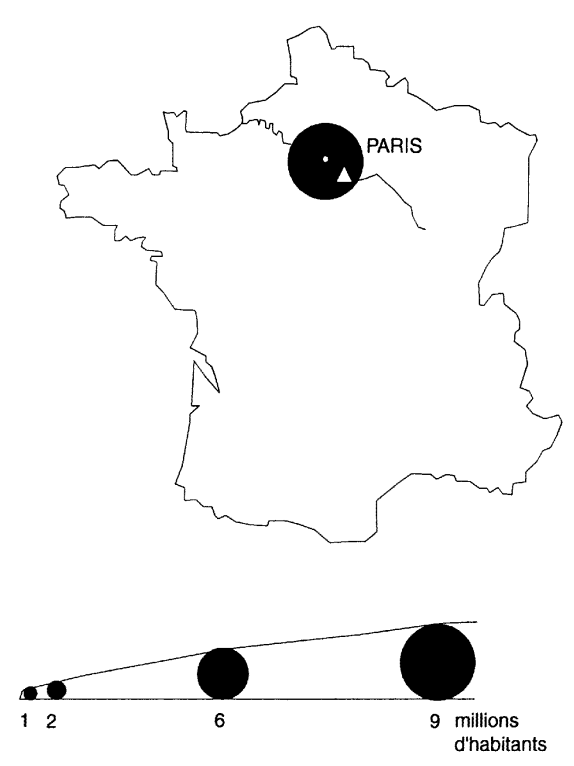
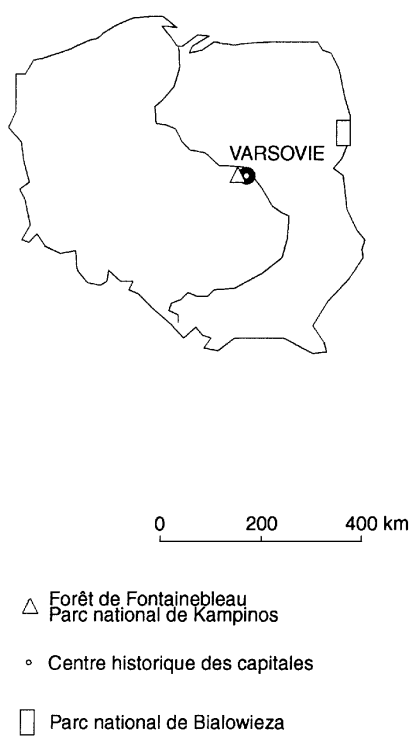

Fig. 1. Deux forêts aux portes de leur capitale nationale. (Sources : F. Moriconi-Ebrard (1994); Atlas (2002).)

Pourquoi comparer forêt de Fontainebleau et parc national de Kampinos, Paris et Varsovie? (Fig. 1). Parce que la démarche comparative est au cœur de la méthodologie des géographes, soucieux de situer l'ici par rapport à l'ailleurs et de dépasser la singularité des lieux pour en tirer des règles plus générales ${ }^{1}$ (Arnould et al., 1997a). Pourquoi la France et la Pologne, alors qu'une multitude d'autres associations à l'échelle de l'Europe peuvent être envisagées? Londres, Berlin, Budapest, Vienne, Genève (Silva, 1997) pourraient légitimement se prêter à ce même exercice d'éclairage réciproque des principes à l'œuvre dans la gestion des espaces et des individus remarquables. Le choix de traiter du binôme franco-polonais se justifie pour des facteurs de parenté écogéographique et de forte ressemblance de certaines structures paysagères. Mais il est aussi conforté par des raisons d'ordre culturel. La Pologne, et surtout ses classes dirigeantes, a toujours eu des liens privilégiés avec la France. Sur le plan historique, Stanislas Leczinski a été duc de Lorraine. Au plan géographique, au nord et au sud de Varsovie, les quartiers de Mokotow et de Joliborz ont une origine toponymique française : ils sont la version slavisée des qualificatifs expressifs de «Mon coteau » et de «Joli bois». Par ailleurs, les forestiers polonais ont été influencés par les deux grandes écoles de sylviculture européennes. La germanique était dominante bien sûr, mais, par réaction, certains forestiers polonais ont prêté une vive attention aux principes de la foresterie française (Devèze, 1965;

\footnotetext{
${ }^{1}$ Matériaux collectés par Caroline Cieslak dans le cadre d'une thèse, interrompue, sur La Gestion de la nature en France et en Pologne: Kampinos et Fontainebleau, et par Paul Arnould, membre du conseil scientifique de la réserve de biosphère du pays de Fontainebleau.
}

Arnould, 1997b). Ces indices signifiants traduisent la forte interaction des deux ensembles. Leur étude conjointe y gagne en légitimité. L'interrogation essentielle qui parcourt ce travail consiste à examiner comment les forestiers sont parvenus à gérer ces forêts pas comme les autres que sont les forêts de proximité des capitales nationales. Les forestiers jouent-ils un rôle actif et conscient dans cette irrésistible focalisation sur les arbres exceptionnels ou résistent-ils - et, si oui, comment - à cette sorte d' «arborimania », typique du monde urbain? (Corvol, 1987; ONF, 1993, 1996, 1997.)

Fontainebleau est une des forêts les plus connues de France et d'Europe. Le premier article, consacré à une forêt, dans la nouvelle revue grand public Forêts magazine, lui est dédié (Arnould et Delfour, 2003). Elle est traversée par l'autoroute A6, dite aussi "du sud» ou "du soleil », une des voies routières les plus fréquentées d'Europe, avec un trafic annuel de plus de 17 millions de véhicules, dont 3 millions de poids lourds (Hermeline et Rey, 1998). Elle offre des paysages spectaculaires, au cœur du Bassin parisien. Â proximité du péage de Fleury-en-Bière, les chaos de grès composent un paysage où la trilogie classique et sage du centre de l'Île-de-France, plaine, plateau et versant, prend une allure de montagne (Arnould, 2002). Les chiffres de visites sont tout aussi éloquents dans leur expansion vertigineuse : 9 millions en 1976 (Ballion, 1975), 13 millions en 1990, 17 millions en 2001, soit le même ordre de grandeur que celui de la circulation automobile au travers du massif (Maresca, 2001). La fréquentation de Fontainebleau éclipse celle d'Eurodisney (13 millions de visites en 2001) et de tous les monuments de la capitale. Même la cathédrale Notre-Dame de Paris, le site parisien le plus visité 


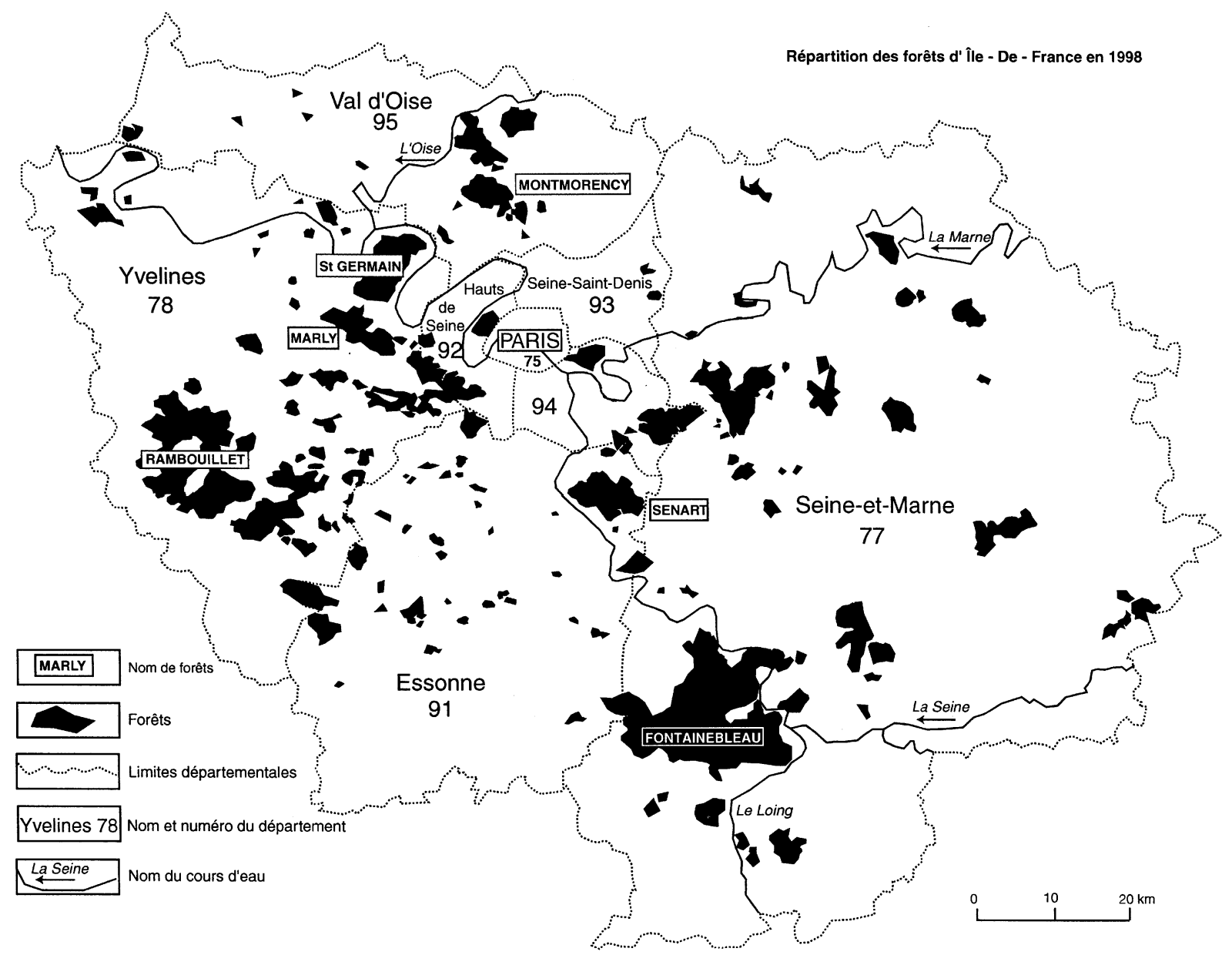

Fig. 2. Fontainebleau, une forêt géante en Île-de-France. (Source : ONF-DR/Sylvétude-LN/JN, 1998.)

(et d'accès gratuit, tout comme la forêt), ne comptabilise que 12 millions de visites par an. Fontainebleau précède largement la tour Eiffel et le musée du Louvre qui n'en attirent qu'environ 6 millions chacun. Le parc national de Kampinos, au nord-ouest de Varsovie, qui n'a pas suscité la même floraison d'études de fréquentation, ne peut rivaliser avec ces chiffres. Pourtant, la comparaison Kampinos/Fontainebleau permet d'amorcer une réflexion sur la place de l'arbre remarquable dans des espaces forestiers périurbains. Le remarquable de France est-il celui de Pologne? Dans les deux pays, le naturel prime-t-il sur le culturel? Le remarquable d'hier est-il encore celui d'aujourd'hui?

Ces deux espaces ont en commun d'être situés à proximité de leur capitale : Fontainebleau à $60 \mathrm{~km}$ de Paris, Kampinos à moins de $10 \mathrm{~km}$ du centre de Varsovie, mais en fait tangent sur son flanc oriental avec le nord de l'agglomération, situation d'extrême proximité, tout à fait originale en Europe (Misiak, 1994) (Fig. 2 et 3). « Le génie de ces lieux », pour reprendre le titre d'un article de Bernard Kalaora (1983), tient-il aux données liées à l'espace et à l'écologie ou est-il fondé sur la présence d'individus que les hommes ont distingués sous le vocable $\mathrm{d}^{\prime}$ « arbres remarquables »? Pourquoi privilégier la perception des espèces à propos d'espaces aussi bien caractérisés? Les arbres remarquables peuvent-ils constituer les emblèmes de ces forêts au voisinage de la ville? L'individu permet-il de mieux parler de la forêt que les allusions aux communautés végétales et aux structures forestières ? L'identification de Fontainebleau avec le chêne Jupiter, dont la mort a provoqué une émotion et des polémiques, savamment entretenues par la presse nationale et régionale, confirme cette hypothèse (Cans, 1994 ; Touzet, 1994). À Kampinos, le "chêne Jagellon», le «chêne des insurgés » (Photo 1) ou le « chêne des combattants » sont aussi les représentants de cette cohorte d'arbres "pas comme les autres » (Fig. 4). Le cas du «pin des insurgés», déraciné depuis une tempête de 1984, mais qui continue de figurer sur les cartes, témoignage toponymique d'un refus de l'acceptation de la mort de l'arbre, est le pendant polonais du «chêne Jupiter » (Fig. 5). Désormais, dans une situation de nouveaux rapports de l'homme à la forêt, la personnalisation des arbres progresse à grands pas.

Dans quels contextes géo-historiques se font ces deux démarches de valorisation de l'arbre remarquable? 


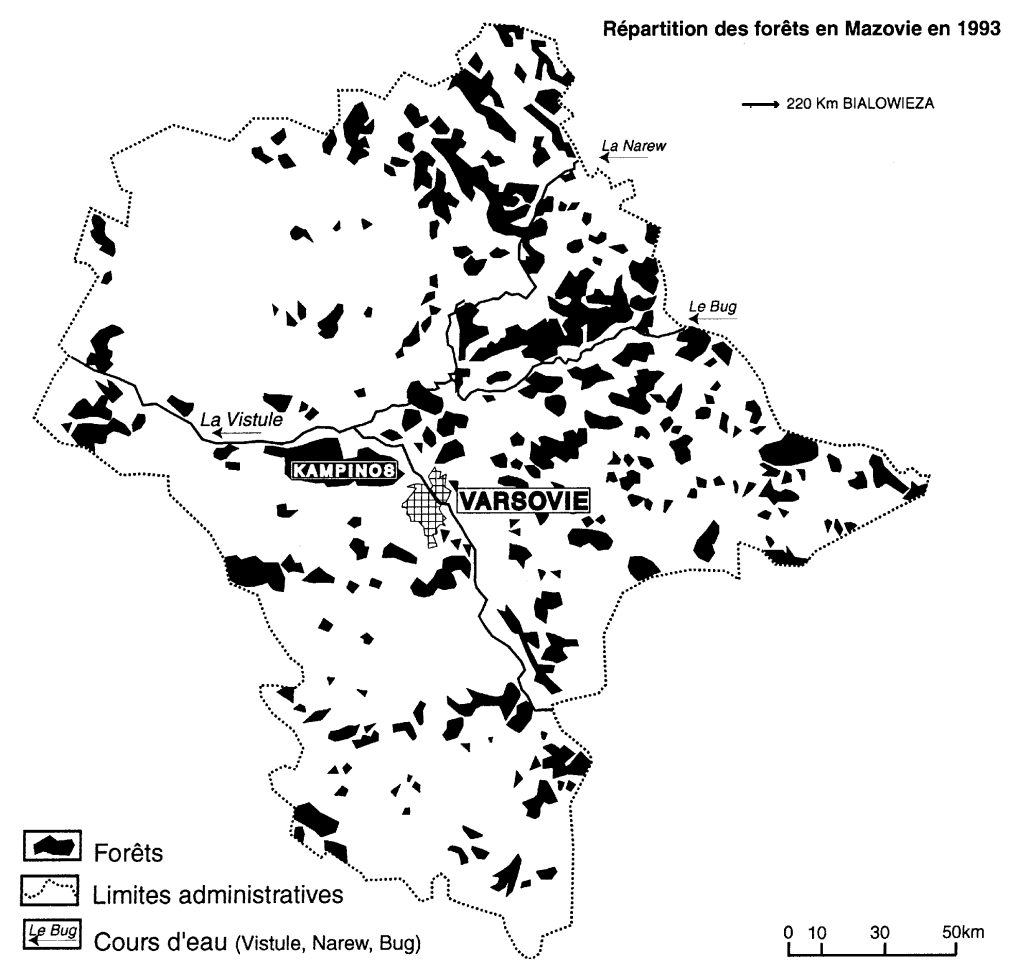

Fig. 3. Kampinos, la plus grande forêt de Mazovie au sud de la Vistule. (Sources : 1. Carte de la protection de la nature, 1/750 000, Instytul Ochrony Srodowiska, Varsovie, 1993; 2. Carte administrative de la Mazovie, Ambassade de Pologne, 2002 (échelle approximative).)

La proximité de la capitale joue-t-elle un rôle pour expliquer ce phénomène en expansion (Arnould, 1997c ; Kalaora, 1981a,b)?

Comment accède-t-on au statut de remarquable? Entre les bio-indicateurs, les marqueurs de territoire, les témoins de l'histoire (ou porteurs d'histoire), les arbres symboles de références culturelles, quelle est la voie royale pour accéder à ce statut de privilégié, repéré par un rond bleu sur le tronc à Fontainebleau ou marqué du signe de l'aigle à Kampinos (Photo 2)?

Quels sont les acteurs qui participent à cette mise en scène de l'arbre au détriment de la forêt ? Peut-on avancer l'hypothèse que ce mouvement de glorification de l'individu participe d'un mécanisme plus général, caractéristique des sociétés urbaines, mais qui puise ses racines dans les mythes et les légendes initiés par les sociétés rurales?

\section{L'utile comparaison. Fontainebleau et Kampinos : $1500 \mathrm{~km}$ de distance, mais un air de famille}

Fontainebleau et Kampinos peuvent être définis comme deux massifs forestiers témoins de la forêt tempérée de façade occidentale du continent européen. Mais leur situation dans ce vaste biome de plaine est cependant sensiblement différente. Fontainebleau, proche du cœur du domaine tempéré, est aussi une forêt aux marges orientales du secteur dit atlantique ou océanique. C'est une forêt de feuillus par excellence (généreusement enrésinée depuis le XIXe siècle cependant). Kampinos, sur les marges nord et orientales du même domaine, est déjà proche de la forêt dite mixte et des prairies de l'Ukraine. Les résineux s'y taillent la part belle, notamment l'omniprésent pin sylvestre (Simon, 2000). Mais, tout autant que ce contexte bioclimatique, la situation des deux forêts à proximité de leurs capitales nationales respectives constitue l'élément structurant décisif (Fig. 1). Leurs passés, en faisant successivement, ou simultanément, des forêts aristocratiques, bourgeoises et populaires, sont également de puissants facteurs de différenciation de ces espaces (Arnould, 1997c; Heymanovski, 1966; Kronika KPN, 1963).

Le statut de Fontainebleau est très ambigu. C'est sans doute une des forêts de plaine les plus "manipulées » par l'homme, mais c'est aussi une des forêts qui évoquent le plus un «milieu naturel». Cela est même vrai chez les scientifiques : elle a ainsi servi de référence, dans le cadre du programme MAB (Man and Biosphere), pour l'étude de l'écosystème de référence des forêts tempérées de plaine : la hêtraie. Cela se confirme dans les travaux de vulgarisation. Dans l'ouvrage coordonné par Antoine Lorgnier intitulé Forêts, une illustration du chapitre sur les forêts tempérées est légendée «... Certains massifs restent cependant presque vierges 


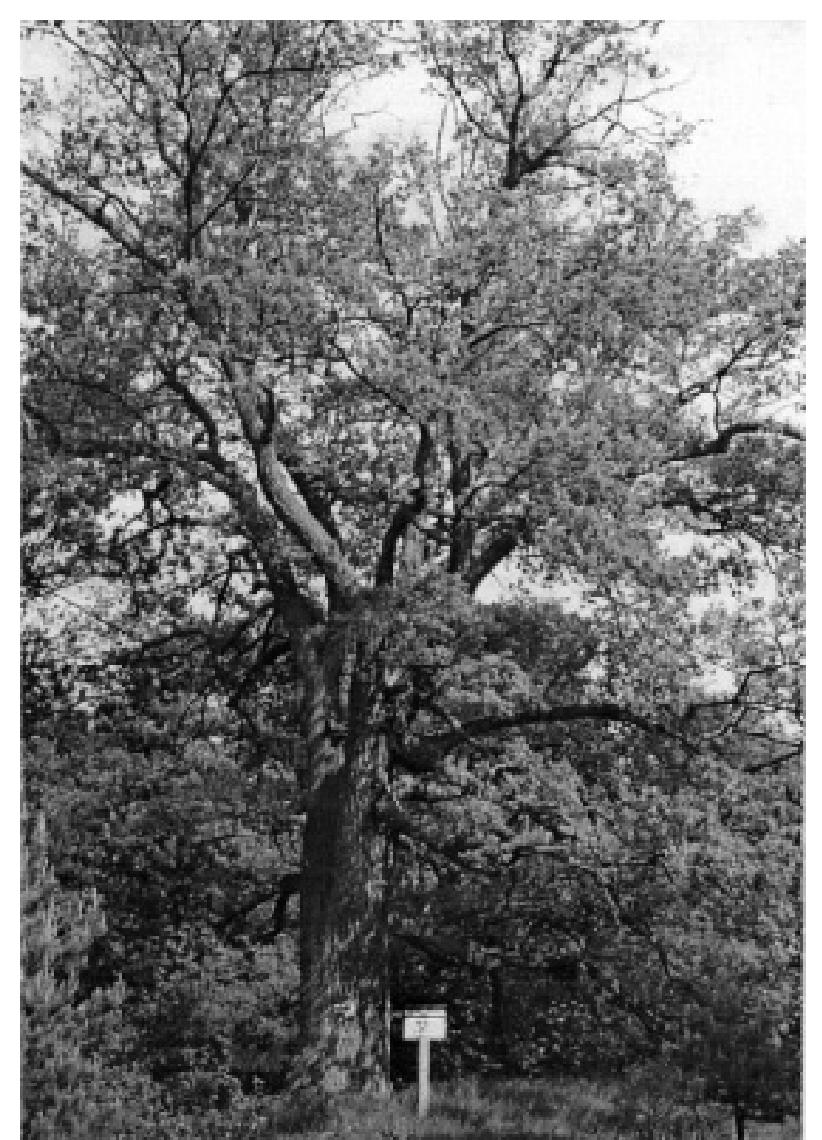

Photo 1. Le chêne des insurgés de 1863 : arbre et spiritualité. Arbre monumental de Kampinos, dédié aux martyrs polonais de la deuxième insurrection contre les Russes en 1863. Il symbolise le mysticisme et le patriotisme polonais. Il voisine avec le pin des insurgés. (Source : photo A. Wierzbicki, vers 1985.)

tels que Fontainebleau en France, Bialowieza en Pologne ou Töfsing en Suède » (Lorgnier, 1992). Même si l'extension à tout le massif du statut de réserve jadis " artistique » et aujourd'hui «biologique » de quelques parcelles (5 à $10 \%$ de la surface totale), qui peut légitimer la qualification "presque vierges », est manifestement abusif, l'homologue polonais de Fontainebleau est donc plus la forêt de Bialowieza que celle de Kampinos. Aux marges orientales de la Pologne, à cheval sur la frontière avec la Biélorussie, Bialowieza est surtout connue par sa célèbre réserve de bisons. Elle témoigne aussi de la luxuriance et de la puissance des forêts mixtes, opposées à la pauvreté et à la simplicité relative de la forêt boréale de conifères (Falinski, 1986 et 1991). Kampinos ne bénéficie pas de la même aura historique, ni de l'extraordinaire capital biologique que recèle Bialowieza, mais sa situation à proximité de la capitale peut compenser ce déficit de biodiversité et de célébrité naturaliste. Le fait que Kampinos soit situé dans le voisinage de l'École d'agronomie et de foresterie de Varsovie en a fait un lieu privilégié d'études et de stages pour les étudiants et les chercheurs.

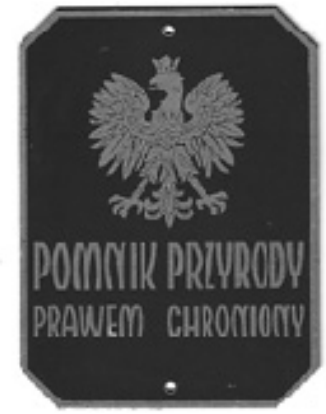

Photo 2. Plaque métallique apposée sur les arbres remarquables en Pologne. Traduction : "monument de la nature, protégé par la loi ».

La forêt de Fontainebleau est dominée par trois essences aux statuts écologiques et historiques fort différents : le chêne, privilégié par l'homme, le hêtre, sans doute l'essence la mieux adaptée aux conditions actuelles de milieu, et le pin sylvestre, propagé par l'homme depuis la fin du XVIII ${ }^{\mathrm{e}}$ siècle. Le pin a connu une expansion tout à fait spectaculaire comme essence pionnière aux côtés du bouleau dans les landes et les pelouses sur sables acides. L'analyse des peuplements forestiers, menée dans le cadre du nouvel aménagement, 1996-2015, aboutit à proposer des chiffres qui traduisent la nette suprématie du chêne parmi le trio des essences dominantes : $42 \%$ de peuplements de chênes dominants, contre $11 \%$ pour le hêtre et $28 \%$ pour le pin sylvestre (Gougis, 2000). Paradoxalement, ces chiffres masquent les réalités des dynamiques forestières : l'essence symbole de Fontainebleau, le chêne, est menacée par un autre feuillu, le hêtre. Les fines études démographiques, dynamiques et écologiques, menées dans les réserves biologiques intégrales par Georges Lemée, dans le cadre du programme MAB (Lemée, 1989 et 1990), et par Micheline Hotyat (2003) le prouvent clairement : le hêtre, essence d'ombre, est en train de supplanter le chêne, essence de lumière, dont la régénération est perturbée sous le couvert sombre des hêtraies. Par ailleurs, dans les pelouses et les landes, le pin sylvestre est un pionnier qui tend à devenir envahissant. La belle étude de Jean Guittet sur la dynamique des pins démontre aisément leurs puissantes capacités à se disséminer et à jouer un rôle majeur dans les stratégies de compétition spatiale entre feuillus et résineux (Guittet et Laberche, 1974). Si, malgré ce fort dynamisme, les résineux ne représentent que le tiers des peuplements à Fontainebleau, il n'en va pas de même en Mazovie (Fig. 3), où les pins sylvestres sont l'essence forestière archidominante (Simon, Mosgawa et Cieslak, 1997).

Les roches en place qui constituent le soubassement de la forêt de Fontainebleau sont des matériaux sédimentaires déposés au centre du bassin parisien au milieu de l'ère tertiaire, à l'oligocène essentiellement, il y a plus de 25 millions d'années. Parmi le feuilletage géologique 


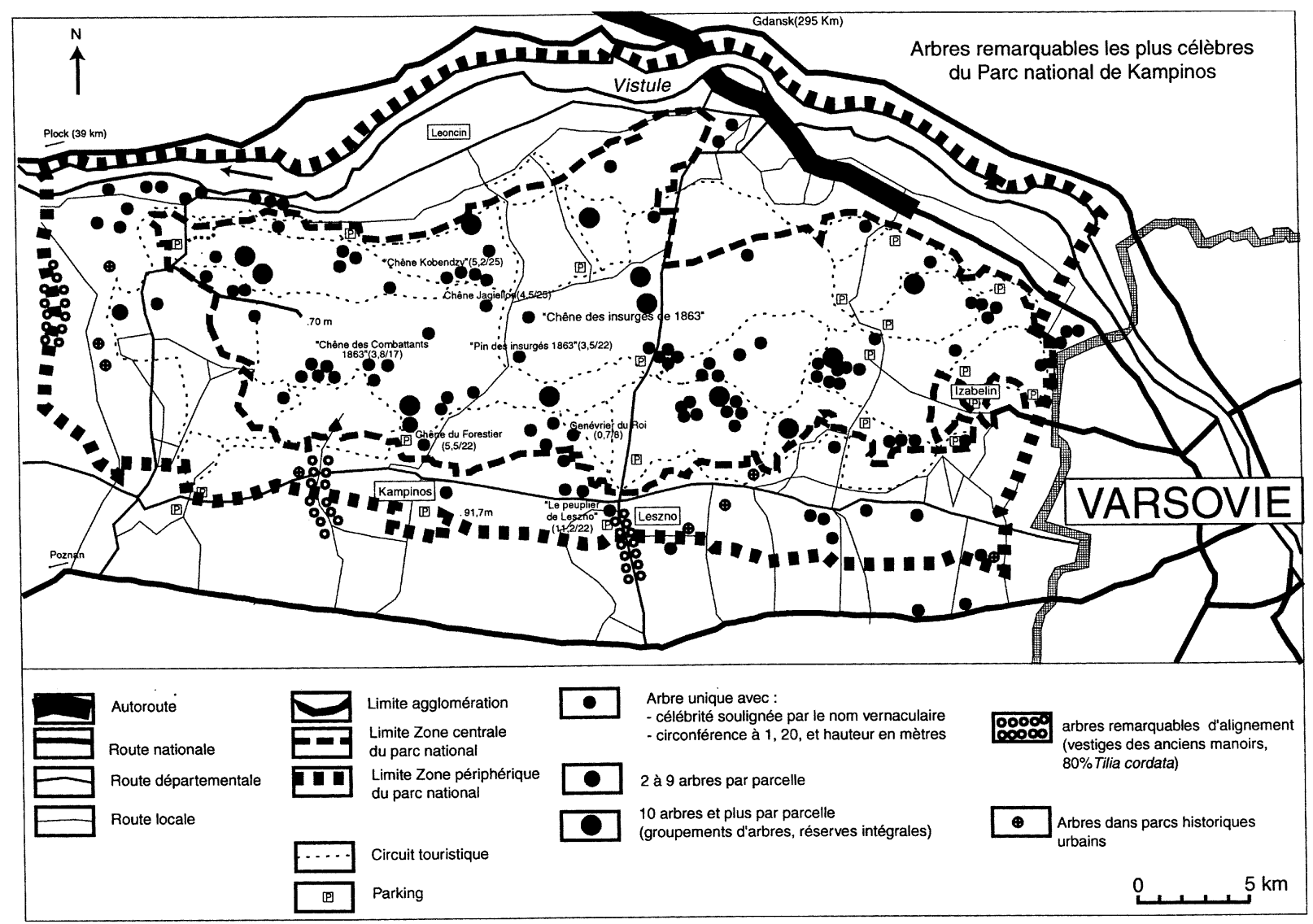

Fig. 4. Répartition des arbres remarquables à Kampinos : un modèle centre-périphérie. (Sources : 1. Mapa topograficzno-turystyczna, WZK, 1/40 000, 1999; 2. Kampinoskiszla k rowerowy, 1/100 000, KPN, 1997 ; 3. Mapa topograficzna, 1/50 000, GGK. Warszawa, 1995 (6 cartes).)

qui fait alterner les calcaires, les marnes, les argiles et les sables, ces derniers jouent un rôle essentiel. Ils s'imposent sur tous les autres matériaux par leur épaisseur, atteignant parfois près de 50 mètres, leur plus ou mois forte cimentation en grès, leurs déplacements et leurs remaniements par les eaux de ruissellement et les vents, les sols de type podzolique qui les caractérisent. À Kampinos, également, le sable est le substrat omniprésent sous la forêt.

Le modelé de Fontainebleau, tout comme celui de Kampinos, est fondamentalement lié à des processus où alternent, suivant les périodes, le rôle des eaux marines, puis continentales, de la glace et du vent. À Fontainebleau, l'énorme masse des sables est d'origine marine. À Kampinos, les sables ont été épandus sur le front des inlandsis qui ont recouvert à plusieurs reprises le Nord de l'Europe lors des glaciations quaternaires. Mais l'essentiel du modelé est lié aux processus d'origine périglaciaire qui ont affecté les deux régions il y a un peu plus de 10000 ans. Les héritages glaciaires et périglaciaires sont décisifs. À Fontainebleau, ils expliquent la présence des matériaux sableux, de granulométrie très fine, en placage superficiel, sur les plateaux calcaires. Seul le vent a pu prélever des sables à nu sur les versants ou dans les fonds des dépressions, en voie d'approfondissement, pour les étaler sur les plateaux environnants. Ces sables sont des éléments écologiques et géographiques majeurs pour la structuration des milieux et des paysages à Fontainebleau comme à Kampinos. Dès que la couverture vivante des plateaux et des versants est déchirée, les sables affleurent à nu et deviennent des proies de choix pour l'érosion hydrique ou éolienne : la ravine, le micro-torrent et la dune sont les formes de relief symptomatiques de ces processus d'érosion efficaces, spectaculaires et spécifiques. Ces sables siliceux très purs expliquent l'omniprésence des sols acides et notamment des podzols qui sont la véritable signature pédologique des deux forêts. Souvent les humus épais, de type mor, forment l'horizon superficiel. Les moindres fosses, les ravines d'érosion, les paquets de sols arrimés aux galettes racinaires des chablis font apparaître la violence des contrastes de couleur, de texture et de structure des podzols. Le feuilletage du noir de l'horizon humifère contraste avec la couche pulvérulente d'un gris cendreux, parfois quasiment blanc, tranchant vigoureusement avec les horizons d'accumulation aux teintes vives, brunâtres, rougeâtres ou noirâtres, à la structure compacte parfois proche du grès. Omniprésence du sable et sols typés acides donnent aux deux 


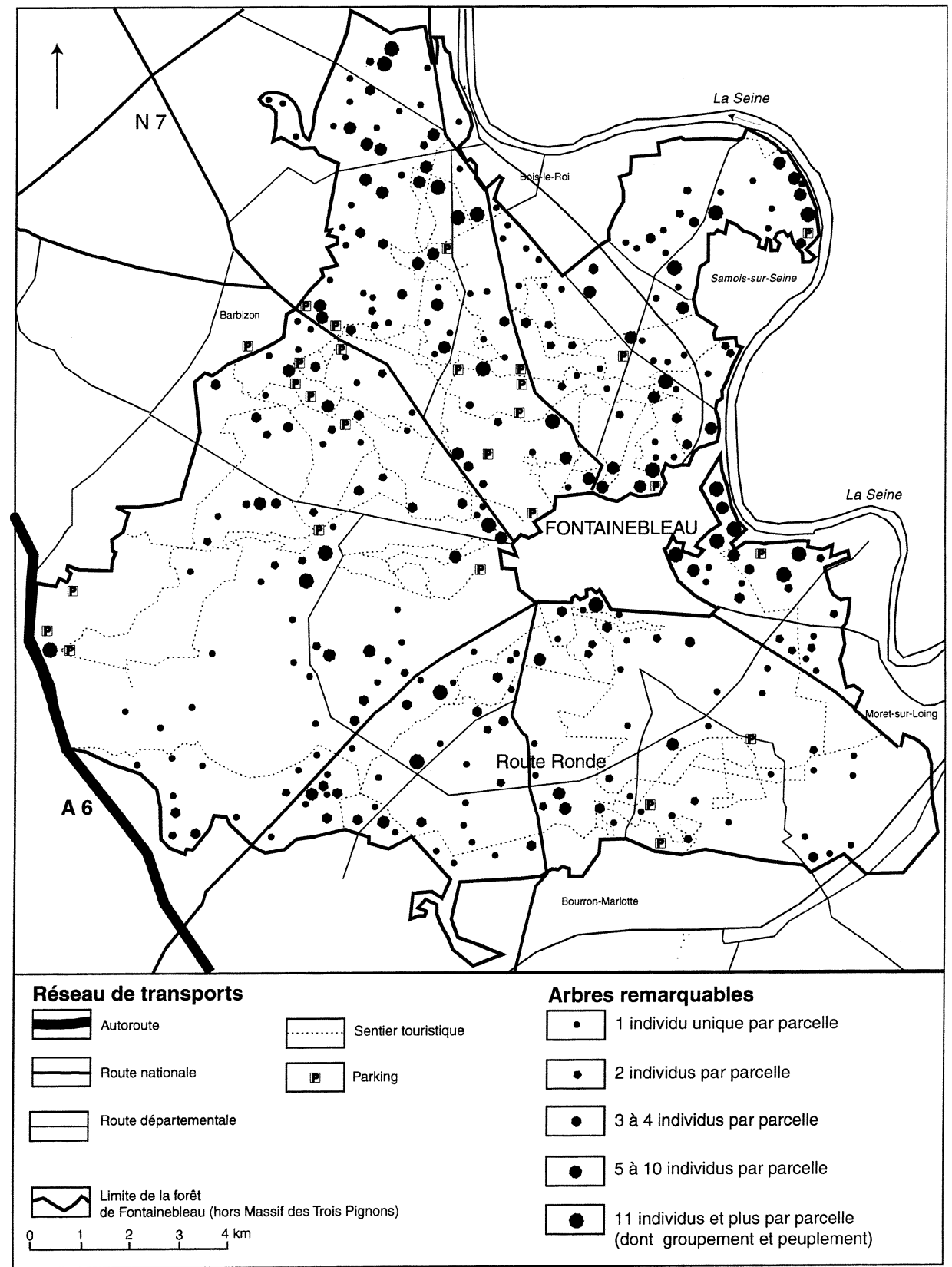

Fig. 5. Localisation des arbres remarquables à Fontainebleau, structures ponctuelles et linéaires : un dispositif spatial peu lisible. (Sources : 1. Carte de la forêt de Fontainebleau, 1/25 000, IGN, 1997 ; 2. AAFF (1998).)

forêts des affinités de substrat qui contribuent à gommer leur éloignement géographique.

Pour comprendre les enjeux, les paysages et les dynamiques de Fontainebleau et de Kampinos, il est nécessaire de les resituer constamment par rapport aux organismes urbains hors normes qui les jouxtent. Paris et Varsovie sont toutes deux des agglomérations millionnaires, mais la comparaison tourne vite court. Parmi les multiples données chiffrées démontrant la suprématie de la capitale française, le fait que la région Île-de-France rassemble près de 11 millions d'habitants, soit $20 \%$ de la population française, sur seulement $2 \%$ du territoire national, confirme le diagnostic formulé au lendemain de la guerre de 1939-1945 par Jean François Gravier, en une formule choc qui marquera les esprits : «Paris et le désert français ». Autant Paris est le symbole de la capitale hypertrophiée, autant Varsovie est une ville parmi d'autres en Pologne (Brunet et Rey, 1996). Si Varsovie est une ville immense par la superficie - 48500 ha dont 24300 ha de sols urbains -, elle n'est pas de taille et de masse impressionnantes à l'échelle de la Pologne. Son agglomération ne rassemble que 1640000 habitants sur les 24 millions de Polonais, soit $5 \%$ de la population du pays. Elle est supplantée par la conurbation de haute Silésie, dans le 
bassin de Katowice, qui en compte 2300000 . L'absence d'un centre urbain hégémonique est une des caractéristiques originales du réseau urbain polonais. Ce polycentrisme urbain peut sans doute aider à comprendre que les questions d'aménagement et d'environnement ne présentent pas en Pologne, dans la région capitale, le même degré d'acuité et de sensibilité qu'en France. De même, Kampinos n'est pas une forêt aussi médiatisée et disputée que Fontainebleau.

Les grands massifs forestiers de la région Île-deFrance ont presque tous le statut de forêts domaniales pour la majeure partie de leur surface. La liste des grandes forêts prestigieuses y est longue : outre Fontainebleau, les noms de Rambouillet, Montmorency, Saint-Germain, Marly, Sénart, Compiègne... rythment la litanie de ces anciennes forêts de chasse royales et seigneuriales, devenues domaniales (Fig. 2). La propriété communale y est faible. Elle est même totalement absente de plusieurs départements de la périphérie parisienne (Arnould et al., 2000).

\section{Du remarquable}

En première approche, définir le remarquable ne paraît pas poser de problèmes insurmontables (Balanger, 1989; Escurat, 1996; Mosnier, 1990; Moigneu et Lecompte, 1997 ; Pourtet, 1979). En fait, le choix des critères mérite discussion. La définition de l'arbre remarquable constitue même un puissant révélateur des perceptions et des représentations de la forêt, à l'œuvre dans le corps social. Tel critère qui semble évident à première vue peut être interprété de façon radicalement différente : ainsi, privilégier le grand et le gros peut vouloir dire mépriser le faible et le chétif ou le «normal» anonyme, en peuplement. Derrière le culte du puissant, il est possible de repérer un eugénisme latent ou une lecture de la société en termes élitistes. Ces interprétations ne sont certes que rarement mentionnées, ce qui ne signifie point qu'elles ne soient pas pertinentes.

« Remarquable » fait partie de ces termes dont la définition est éminemment subjective, comme «beau » et «rare». Frédérique Lecomte (2001), dans le petit guide de gestion de l'ONF, issu d'un gros travail de réflexion collective, intitulé Les arbres remarquables en forêt, esquive habilement le problème de la définition par une pirouette : "Trop subjectif, trop compliqué, trop inutile... . écritelle à propos de ce qu'elle considère comme un travail un peu futile. Premier réflexe compréhensible d'ingénieur et de gestionnaire, soucieuse d'efficacité, mais qui ne saurait satisfaire le chercheur en sciences humaines. Comprendre la façon dont l'arbre remarquable est défini est tout sauf inutile à nos yeux. C'est au contraire un acte lourd de sens et de significations dans le choix des termes, les références utilisées, les comparaisons proposées, les exemples utilisés. S'abstenir de définir, c'est implicitement se ranger dans une catégorie d'utilisateurs refusant le débat théorique, tenant pour négligeables les inquiétudes épistémologiques et les interrogations sémiotiques et sémantiques.

Pourtant, après avoir récusé en apparence le travail de réflexion sur la définition, Frédérique Lecomte se livre à une fine analyse des différentes acceptions de la notion d'arbre remarquable. Elle s'appuie notamment sur celle, proposée par deux experts, Robert Bourdu et Michel Viard (1988), qui caractérise bien le caractère elliptique et anthropomorphique de toutes les définitions : "L'arbre remarquable n'est pas seulement un arbre d'élite que sa taille ou sa forme distingue de tous les autres. C'est aussi l'arbre consacré par une histoire et qu'une collectivité humaine reconnaît comme élément de son patrimoine. » Elle nous propose différents registres de qualificatifs qui situent bien le remarquable du côté de l'exceptionnel lorsque l'on parle d'arbre «d'élite», d'arbre "plus » ou d'arbre «d'avenir ». "Élite» fait référence à des attributs de sociabilité, à des qualités supérieures à la moyenne et évoque une appartenance à une minorité. «Plus » qualifie des performances spatiales remarquables en termes de taille, de diamètre, de volume, donc de productivité et de biomasse, de croissance et de développement. " Avenir » est du registre temporel, le terme projette dans le futur, évoque des promesses de rendement, des potentialités hors norme, une possibilité de créer des lignées hors du commun.

Que ce soit sur le plan social, spatial ou temporel, les mécanismes utilisés pour nommer les arbres remarquables par le forestier sont ceux de la distinction. Une des autres caractéristiques majeures des procédés de nomination, dans le langage plus populaire, retrouve les mêmes catégories que celles utilisées par les forestiers. Elle consiste à utiliser des références anthropomorphiques ou historiques. Au premier cas appartiennent les vocables faisant référence à l'âge, à la vieillesse, comme "l'ancêtre », " le Mathusalem », ou encore à la taille, comme «le géant», ou enfin à des comparaisons politiques, «le roi » ou «le président». Avec ces noms identifiant dans un massif soit les plus vieux, soit les plus hauts, soit les plus puissants, on retrouve les mêmes registres de qualification mobilisant le temps, l'espace et le social.

Derrière ces critères de la distinction se profile hélas, selon nous, un risque majeur, celui d'un eugénisme latent. Le véritable culte de l'exceptionnel, le mythe $\mathrm{du}$ record, la course au plus grand, au plus gros, au plus vieux semble disqualifier tous les arbres de l'ordinaire. Exit le banal, le quelconque, le commun (Photo 3)! Seuls comptent l'admirable, le formidable, l'inoubliable, le considérable! Cette tentation de privilégier une race supérieure d'arbres est curieusement compensée par le fait que peuvent accéder au statut d'arbres remarquables les individus difformes (Photo 4) : le nain (le syndrome 


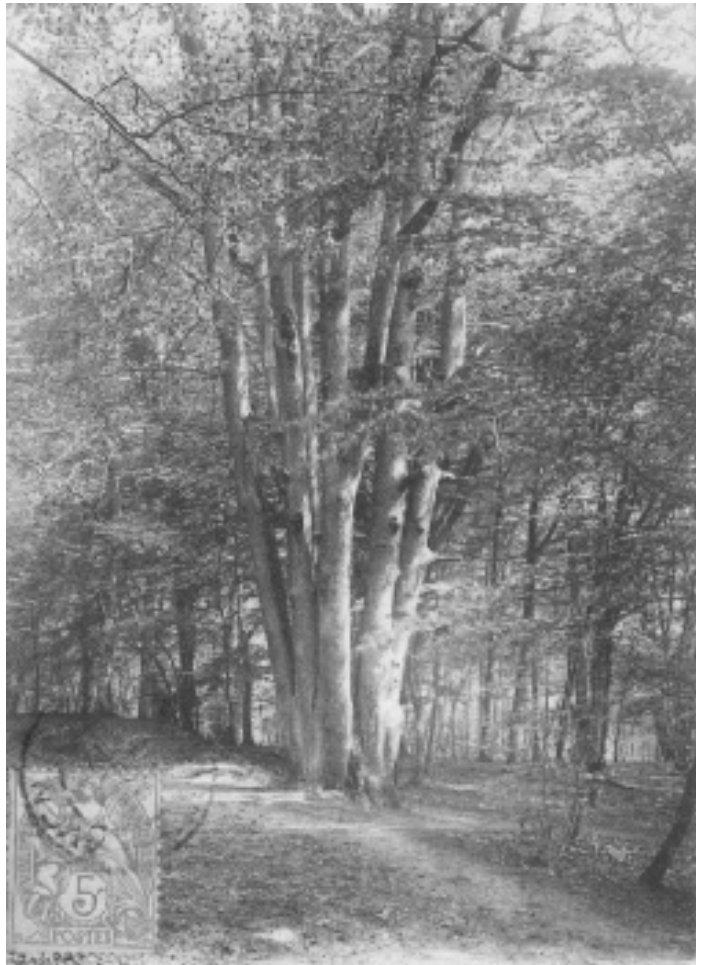

Photo 3. Le bouquet du Nid d'aigle : l'arbre objet de curiosité. Selon C. Colinet : "Le bouquet du nid d'aigle est composé de dix arbres partant de la même souche et s'élevant hauts et droits comme les épis d'une gerbe gigantesque». (Source : C. Colinet (sans date), Collection de la bibliothèque municipale de Fontainebleau.)

du bonsaï), le tordu (faux de Verzy, dans le parc naturel régional de la Montagne de Reims), le disproportionné, le pleureur, le penché, le boursouflé ne sont pas, loin de là, systématiquement disqualifiés (Rumelhart, 1999). Il n'empêche que la logique de l'arbre remarquable est une logique de l'anormalité. Le moyen, le médian, le commun, le classique sont écartés de ce statut réservé. Cette logique du hors-norme est celle qui est à l'œuvre dans les médias où ce qui «se vend » le mieux est le sensationnel, le catastrophique, l'exceptionnel.

\section{De l'espace, de la nature, de la culture}

La cartographie de la répartition des arbres remarquables à Fontainebleau laisse perplexe le géographe à la recherche de structures spatiales bien identifiées (Fig. 5). Ni les données naturelles, pourtant spectaculaires, avec les vigoureuses oppositions topographiques entre plateaux calcaires (les monts), ou gréseux (les platières) culminant vers $120 \mathrm{~m}$ et les dépressions, à $70 \mathrm{~m}$ d'altitude, ni les grands alignements est-ouest, ni les infrastructures de circulation, voies de transit nord-sud ou route Ronde, ni les phénomènes de proximité des villes emblématiques que sont Fontainebleau ou Barbizon ne

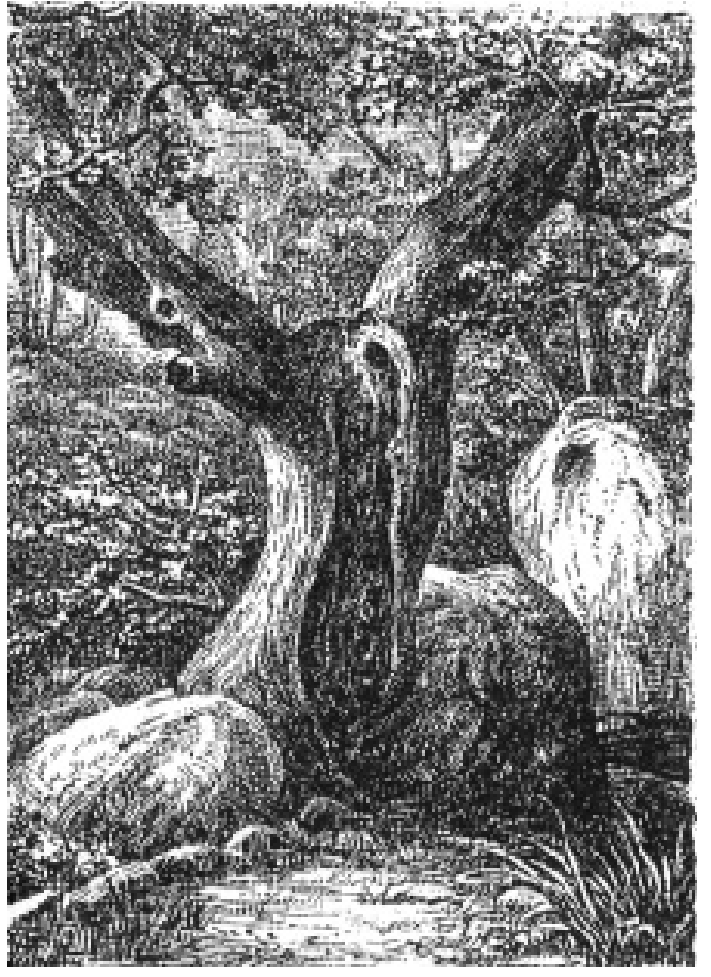

Photo 4. Le chêne François $\mathrm{I}^{\mathrm{er}}$ : la valorisation de la difformité. Gravure du chêne François $\mathrm{I}^{\mathrm{er}}$, ancien arbre remarquable de Fontainebleau qui présentait «un entre creux et tout vermoulu déchiré en ogive». (Source : C. Colinet (sans date), Collection de la bibliothèque municipale de Fontainebleau).

donnent des clés de lecture satisfaisantes. Le semis des arbres remarquables apparaît fortement aléatoire, sans clé de lecture apparente. Il n'obéit pas visiblement à un modèle centre-périphérie. Curieusement, les sentiers ne sont pas des lieux de répartition privilégiés des arbres remarquables (cf. extraits des cartes parcellaires au 1:25000 in AAFF, 1998). Cette répartition est-elle donc liée au hasard ou répond-elle à un souci des «faiseurs d'arbres remarquables » de ne privilégier aucun lieu? Sans souscrire entièrement à cette dernière hypothèse, on peut penser que l'ancienneté de la mise en œuvre d'un système de singularisation a sans doute contribué à ce résultat, étonnant en apparence, d'une répartition plutôt homogène des individus remarquables. Les choix de plusieurs concepteurs se sont en effet superposés ou additionnés. Tout d'abord Claude-François Denecourt, le précurseur (1788-1875), identifiant une centaine d'arbres remarquables dans ses premiers guides (1854), a véritablement fabriqué une mise en scène (en paysage) des arbres, mais aussi des blocs rocheux et des sites, le long de ses parcours «mythologiques ». Son œuvre sera poursuivie et amplifiée par Charles Colinet (1839-1905), avec 350 arbres répertoriés dans le dernier guide de 1931, puis reprise par Pierre Bois et Jean Vivien, avec 700 arbres distingués, de 40 espèces différentes (AAFF, 1988), 
et enfin perfectionnée par l'Association des amis de la forêt qui aboutit à choisir 935 sujets, de 80 essences différentes, sur les huit millions d'arbres de Fontainebleau (1998). Dans un des milieux les plus contrastés de l'Îlede-France, le paradoxe du « gommage » des oppositions contribue à faire de toute la forêt un espace où le remarquable peut être partout présent.

La logique de répartition des arbres remarquables à Kampinos fait plus songer à un modèle centre/périphérie (Fig. 4), alors que, paradoxalement, les structures topographiques et géomorphologiques sont fortement influencées par les grands alignements dunaires à orientation dominante est-ouest. Le dispositif de répartition des arbres remarquables ignore ces fortes contraintes d'ordre naturel, montrant bien que, là aussi, le remarquable est plus de l'ordre du culturel et du social que du naturel. Les parcs des châteaux périphériques et les grandes allées constituent les lieux du remarquable plus que le massif forestier proprement dit. Faut-il y voir aussi le reflet de la combinaison d'une structure sociale fortement élitaire sur les marges avec la puissance, au cœur du dispositif, des scientifiques et des forestiers qui s'attachent plus, dans la logique des travaux phytosociologiques, si importants en Europe centrale, à privilégier l'herbe au détriment de l'arbre? Il est certain que le classement des arbres remarquables à la périphérie du parc permet aux forestiers polonais de contrôler partiellement les propriétaires privés (Zestawienie..., 1996). Le centre de la forêt est ainsi un espace où les arbres remarquables sont relativement rares. En revanche, la périphérie proche de Varsovie est le lieu de la distinction des arbres remarquables, au statut plus privé que public (Arkusz..., s.d.).

Le pouvoir de nommer les arbres remarquables n'est pas un acte insignifiant. C'est une démonstration de contrôle du territoire de façon tout à la fois symbolique et concrète (Arnould, 1986). Les ronds bleus sur les arbres, qui irritent certains puristes, à Fontainebleau, prouvent bien que l'appropriation des arbres par les faiseurs de guides ou par une association est, en fait, un enjeu de pouvoir non négligeable. Les Amis de la forêt de Fontainebleau l'ont bien compris qui renforcent leur légitimité, au côté des forestiers, en manifestant un registre de compétence différent de celui de la technique sylvicole des ingénieurs, mais en relation directe avec les enjeux sociaux d'accueil, si importants à Fontainebleau. À Kampinos, le marquage par l'aigle à deux têtes a plus d'allure que la simple tache bleue de Fontainebleau (Photo 2). La puissance de l'administration forestière polonaise s'y affirme clairement (Andrzejewski, 1994), tout comme le poids des symboliques nationalistes, si fortes dans un pays dont l'identité a souvent été menacée par de puissants voisins hégémoniques (russes, autrichiens, prussiens).

La consultation du tableau comparatif des proportions feuillus/résineux à différentes échelles spatiales, de l'échelon national au massif forestier, réserve des surprises (Fig. 6). À Fontainebleau, les résineux tiennent une place objective à peine supérieure à leur statut national : $39 \%$ pour une moyenne française de $36 \%$. Le massif est pourtant un haut lieu de la contestation antirésineuse, depuis le temps de l'école de peinture dite de Barbizon, où les peintres se faisaient un devoir de déraciner les pins sylvestres, pour les apporter tels des trophées, le soir, à leur quartier général de l'auberge du père Ganne, devenue célèbre depuis lors. Parmi les arbres remarquables, les feuillus sont incontestablement privilégiés (Fig. 7). Les deux chênes, sessile et pédonculé, occupent les deux premières places, talonnés par le feuillu précieux le plus coté financièrement, l'alisier torminal en troisième position. L'alisier de Fontainebleau, espèce emblématique du massif, à défaut d'en être une endémique certaine, occupe la sixième position, incontestablement survalorisée en regard de son poids en termes de superficie ou de nombre d'individus. Les résineux, tant décriés, font plus que de la résistance, avec $23 \%$ d'arbres ayant droit au statut de remarquable. Le pin noir et le pin sylvestre fournissent les mêmes contingents d'arbres remarquables. En septième position, le pin maritime complète le trio de résineux qui ont droit de cité dans les guides successifs établis depuis le milieu du XIXe siècle. Les pins « pittoresques » ont eu, il est vrai, une place de choix dans les tableaux des peintres paysagistes de Barbizon

À Kampinos, dans un contexte où le refus des conifères ne s'est jamais manifesté de manière virulente, l'ostracisme frappant les résineux, qui ne parviennent à accéder au statut de remarquable que de façon exceptionnelle, est tout à fait spectaculaire. Les 72,5\% de résineux du massif, fort proches des $75 \%$ nationaux, contrastent violemment avec le petit $1 \%$ de résineux sortant de l'anonymat (Fig. 4). Si le discours ne disqualifie pas les résineux, leur extrême marginalisation de fait est tout à fait étonnante. Seuls quelques pins sylvestres et de rares mélèzes figurent au hit-parade des arbres-mieux-que-les-autres. Chez les feuillus, la suprématie du tilleul est tout à fait impressionnante. Représentant les trois quarts du stock d'espèces remarquables, il relègue loin derrière chêne, frêne et peuplier. L'orme lisse ne joue qu'un rôle de fairevaloir sur le même plan que le mélèze d'Europe.

Ainsi donc les résineux décriés en France et spécialement à Fontainebleau ont trouvé grâce auprès des « faiseurs d'arbres remarquables ». À l'inverse, en Pologne, pays de couvert résineux par excellence, le résineux singulier est totalement marginalisé tandis que le feuillu remarquable est survalorisé, à Kampinos en particulier. Le culturel l'emporte sur le naturel de façon incontestable. Comprendre les raisons de ces proportions étonnantes n'est pas une mince affaire : héritage de choix historiques, bien entendu, mais sans doute aussi intervention de mécanismes sociaux subtils, contribuant à valoriser certaines essences, considérées comme exceptionnelles, 


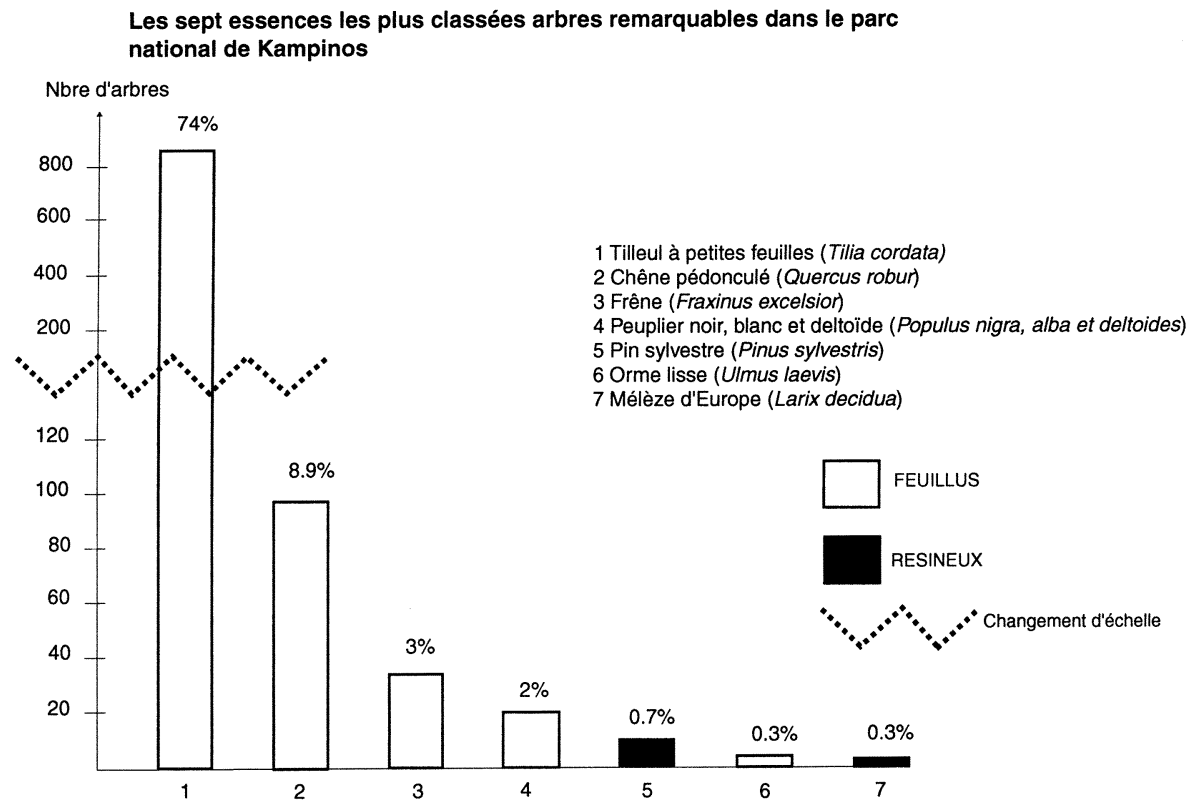

Les sept essences les plus classées arbres remarquables à Fontainebleau

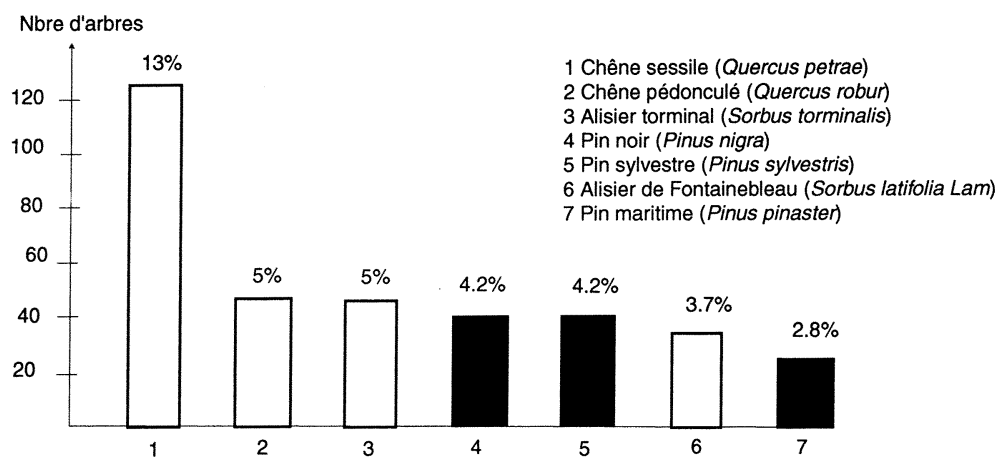

Fig. 6. Arbres remarquables à Fontainebleau et Kampinos. (Sources : Parc national de Kampinos (1996) ; AAFF (1998) ; Dumé (1989).)

ou au contraire à en disqualifier d'autres, jugées trop ordinaires.

\section{De la mort, de la symbolique, des conflits}

Que ce soit à Fontainebleau ou à Kampinos, la mort des arbres remarquables, le Jupiter ou le Jagellon, est vécue, par certains, comme un véritable traumatisme. Que cache cet apitoiement sur la mort de l'arbre? Nous l'interprétons comme un syndrome du mal-être des gens des villes en quête de racines (Urbain, 2002). Les ruraux n'ont pas ce genre d'état d'âme. L'arbre est pour eux fondamentalement un objet fonctionnel. Il peut servir, vivant, au bornage des forêts, ce que l'on appelait jadis les pieds corniers. Lorsqu'il a un port et une taille qui le distinguent de l'ordinaire, il peut jouer le rôle de repère. Mais, par-delà ces usages nobles, sa fonction essentielle est de fournir du bois d'œuvre ou de chauffage. Pour cela l'arbre est taillé, élagué, étêté, émondé sans vergogne.
Il arrive aussi que la mort de l'arbre soit mise en scène, sans excès de sensiblerie. L'abattage des "sapins présidents » des forêts noires, les «joux » du Jura, donne lieu à un cérémonial de fête où se mêlent, certes, une pointe de respect, mais aussi une claire conscience du caractère mortel et éphémère de ces arbres si exceptionnels soientils. Leur remplacement est prévu, programmé, accepté. Autre modèle de rapport au plus grand, au plus gros et au plus vieux des êtres vivants.

En revanche, l'arbre distingué, nommé, symbolisé comme être exceptionnel ne peut plus subir le sort commun de ses congénères, ni même celui auquel il était préalablement voué (anciens têtards «émancipés »). Il a droit au respect. Il mérite l'éternité ou tout du moins de mourir de mort naturelle, et surtout pas sous les coups de hache, de scie ou de tronçonneuse. Cette conception idéologique, aux racines et aux ressorts ambigus (Harrison, 1992 ; Arnould, 1997d ; Dumas, 2002), justifie l'acharnement thérapeutique qui vise à maintenir les arbres remarquables en vie quoi qu'il en coûte, envers 


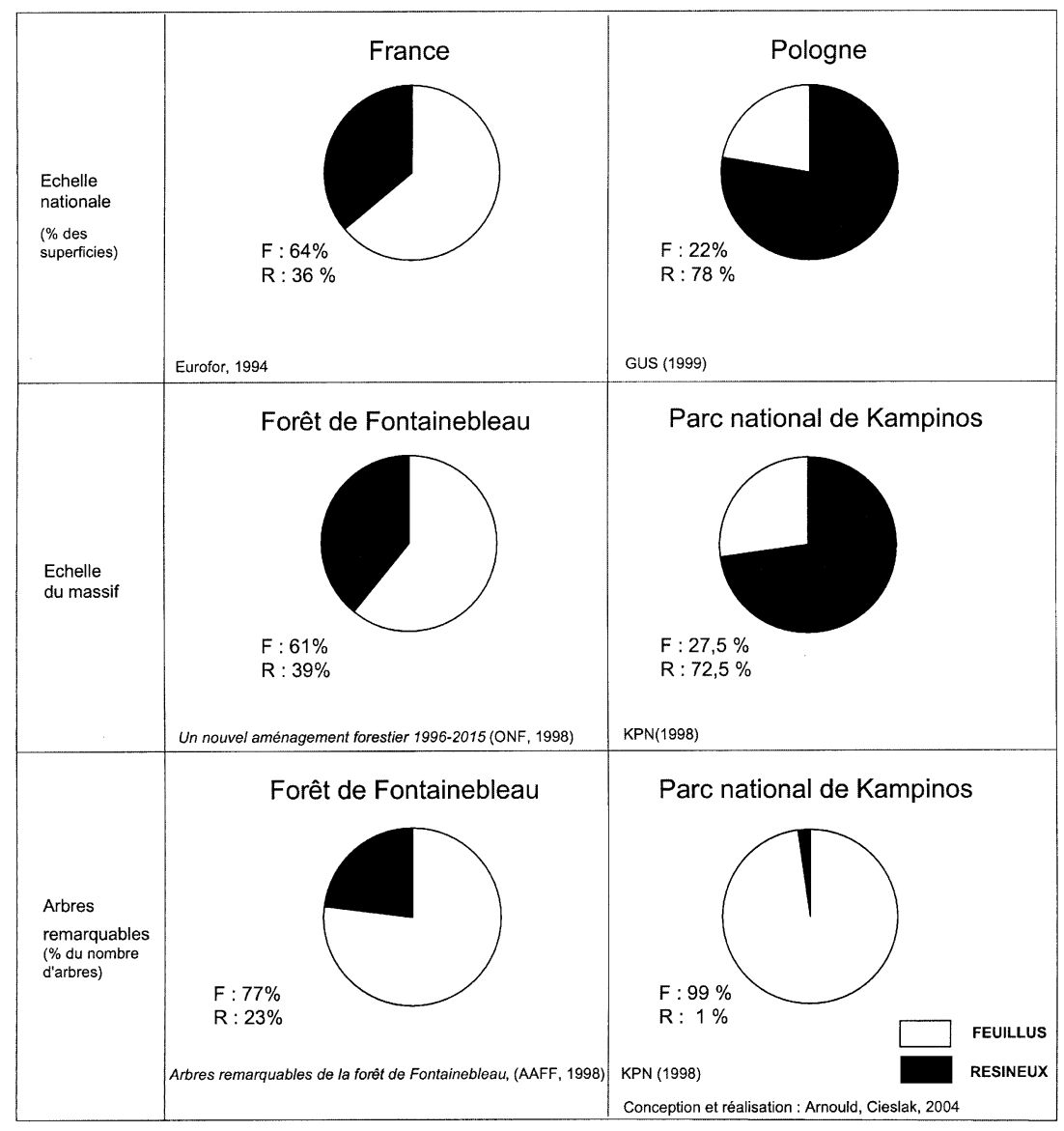

Fig. 7. Feuillus, résineux : du national au local.

et contre tout. Les chirurgiens de l'impossible n'hésitent pas à bétonner les arbres creux, à haubaner les branches prêtes à tomber ou à leur adjoindre de gracieuses béquilles pour les soutenir dans leur immobilité. La pose de broches complète cet arsenal d'interventions aux coûts souvent mirobolants. Si ces techniques de rafistolage ne sont plus pratiquées en forêt, elles restent très présentes en milieu urbain. Nous en avons vu de multiples exemples dans des contextes différents, en pays de Caux, en banlieue parisienne, tout aussi bien qu'à Rome ou qu'à Séoul.

Il arrive aussi que l'on mette le vieil arbre remarquable dans un enclos, tout comme l'animal dangereux est mis en cage, pour que le public puisse le contempler sans s'en approcher. Il ne faut pas que ses branches mortes s'écrasent sur les promeneurs innocents et inconscients.

La gestion de l'arbre remarquable débouche bien souvent sur de redoutables problèmes de légitimité. Peut-on laisser la gestion de cet objet hybride, lourd de nature mais pétri de culture, aux seules mains des forestiers? Les ingénieurs et les techniciens spécialistes en sylviculture, surtout armés pour raisonner en termes de volume, d'accroissement, de productivité, sont-ils les mieux placés pour intégrer les dimensions historiques, sociologiques, symboliques et psychologiques qui sont intimement liées à l'arbre? Ne faut-il pas mieux laisser s'exprimer les scientifiques? Et quelle part laisser aux usagers de la forêt?

À Kampinos, les forestiers contrôlent l'espace forestier du parc national ainsi que la gestion des sujets remarquables. Leur légitimité n'est guère remise en cause pour l'instant. Paradoxalement, dans le cas de Varsovie, une majorité d'arbres remarquables se trouve avoir un statut privé. Ils peuplent les allées et les parcs des châteaux de l'ancienne noblesse polonaise. La question de la gestion de l'arbre remarquable par la sphère privée ou publique n'y est pas encore un objet de débat, alors qu'il $\mathrm{y}$ a là potentiellement, au minimum, une source de comparaisons, voire des logiques différentes, susceptibles de s'affronter. Dans la forêt publique, l'arbre remarquable serait un bien commun apparemment gratuit, dans la forêt privée il pourrait devenir une valeur marchande, un objet d'appel, une marchandise touristique, un produit de consommation naturelle et culturelle.

À l'inverse, à Fontainebleau, les forestiers sont la cible de toutes les contestations possibles et imaginables. Depuis plus d'un siècle, que ce soit avec les célèbres peintres de Barbizon mais aussi avec les populations 
« laborieuses et dangereuses » de charbonniers et de carriers, ils doivent composer, négocier, expliquer, se justifier, vis-à-vis d'acteurs de plus en plus nombreux, qui mettent en cause leur légitimité. La gestion de Fontainebleau est ainsi jalonnée d'actes de contestations où des arbres remarquables par leur âge ou leur origine servent de supports aux débats portant sur la gestion de la plus emblématique des forêts périurbaines de France (Polton, 1995; Vallaeys, 2000).

Ces conflits peuvent être feutrés mais néanmoins virulents, comme avec l'Association des amis de la forêt de Fontainebleau. Récemment, ils ont porté sur l'extension des surfaces en réserves biologiques intégrales. Dans une luxueuse plaquette en couleur, l'AAFF affirme que des réserves intégrales ne sauraient justifier des mesures draconiennes d'interdiction de fréquentation. Ils reconnaissent que « la protection de certains milieux ou de certaines espèces identifiées comme remarquables nécessite parfois une adaptation particulière des règles de gestion ». Ils militent activement pour des réserves biologiques dirigées.

Ils peuvent devenir violents, comme face aux « écoguerriers ", arracheurs de plants de cèdres et saboteurs de matériel forestier. Ces contestataires aux effectifs restreints, pâle mais néanmoins embarrassante copie des «Ecowarriors » britanniques, ont des positions teintées de radicalisme. Leurs actions spectaculaires et parfois violentes, toujours soigneusement mises en scène, fournissent aux médias des sujets croustillants dont ils sont friands. Leur choix de causes symboliques comme la création d'un parc national à Fontainebleau, appuyé sur un groupe de soutien, où les académiciens côtoient les prix Nobel, où les universitaires voisinent avec les artistes, est susceptible de leur valoir un fort courant de sympathie. Quoi qu'il en soit, la gestion de Fontainebleau est une gestion négociée. La chose n'est pas aisée. Les forestiers sont sur le qui-vive. Leurs moindres faits, gestes et écrits sont épiés, épluchés et bien souvent contestés. Pour mieux assurer ce rôle de gestionnaire principal, mais pour s'entourer de garanties, les forestiers de 1'ONF ont réussi à faire participer les usagers et les scientifiques à des structures de gestion originales. La gestion négociée n'est pas un chemin semé de roses. Elle est un véritable combat, continuellement repris, où les alliés d'un jour peuvent devenir les adversaires d'un autre jour.

Traiter tout à la fois de l'arbre, de la forêt et de la ville constitue un exercice périlleux tant le champ des références est vaste. $C^{\prime}$ est cependant un enjeu scientifique majeur dans la mesure où le destin de l'arbre et de la forêt se joue désormais sur la scène urbaine plus que dans les territoires uniquement forestiers. Prendre deux massifs forestiers comme objet permet de mieux débusquer certains des mécanismes à l'œuvre dans la sacralisation de l'arbre en domaine périurbain.

Il est possible de poser comme hypothèse que l'arbre, plus que la forêt, est devenu un véritable « objet intermédiaire ». Sous ce qualificatif, nous entendons un objet qui est par excellence un enjeu dans les négociations concernant les territoires. Son statut ambivalent ou, mieux, polyvalent de produit matériel, d'objet économique mais aussi de bien symbolique, chargé de multiples représentations et significations, lui donne une charge affective propice au dialogue mais aussi au conflit. L'arbre laisse rarement indifférent. Ce n'est jamais un objet neutre. Il oblige à se dévoiler. Il sert de révélateur pour toutes les ambiguïtés liées à un monument naturel. L'arbre, tout autant que la forêt, s'invite au cœur du débat social.

La logique de l'arbre remarquable et celle de l'espace forestier sont fondamentalement différentes. Leur gestion conjointe n'est pas chose aisée. La forêt est un espace fermé, opaque, généralement sombre, difficile à territorialiser, c'est-à-dire à s'approprier sur le plan foncier tout autant que sur le plan symbolique : qui est en effet capable de parcourir tous les sous-bois de long en large, hors des sentiers battus? Bien peu de gens en fait. Le garde forestier chargé d'un triage plus que l'ingénieur absorbé par ses tâches de bureau; le braconnier plus que le visiteur attaché à un itinéraire éprouvé dont il déteste dévier. . À l'inverse, l'arbre est un objet visible et lisible avec lequel il est possible de tisser des liens personnalisés. La forêt est un collectif, une foule, une masse. L'arbre est un individu, une personnalité, une exception. La foule ne fait guère rêver. Elle fait même souvent peur. Au contraire, l'individu isolé invite à l'identification, à la personnalisation, à la complicité.

S'engager dans une politique de distinction des arbres remarquables, c'est en grande partie sacrifier progressivement le naturel au profit du culturel. Le culte de l'arbre remarquable va de pair avec la dépréciation du massif, de la parcelle, de la surface, de l'aréal et la valorisation du ponctuel, du précisément localisé et repéré sur les panneaux de signalisation et sur les cartes. Alors que la parcelle n'a droit qu'à un numéro anonyme, le choix du nom de l'arbre remarquable est une opération à forte charge historique et symbolique. Substituer aux limites plus ou moins floues des mégaparcelles de dix à vingt hectares des lieux précisément identifiés, oblige le gestionnaire à s'engager dans une nouvelle logique d'aménagement où les pratiques classiques vont devoir composer avec le culte de l'imaginaire.

Gérer les arbres remarquables n'est pas une sinécure. Pour le forestier, c'est tout à la fois un honneur et un cassetête. Ces individus prestigieux sont des sujets de gestion de plus en plus tyranniques. Ils échappent au lot commun. Ils obligent à des changements radicaux de conception. Il faut leur réserver un traitement de faveur. Un seul individu prend tout autant d'importance qu'une série de parcelles de plusieurs centaines d'hectares. L'arbre " pas comme les autres » accède au même statut que le milieu rare. Il acquiert une importance équivalente à celle de la 
mare acide, de la pelouse calcicole ou de la lande sèche. . . Il a droit à des chapitres dans les livres, des articles dans la presse, des fascicules personnalisés, sa carte postale, des panneaux le localisant ou le décrivant. Il peut même, honneur suprême, devenir logo. Il en devient alors intouchable. Alors que la plante rare et menacée ou le milieu de haute qualité écologique est d'abord l'objet de référence des écologistes, l'arbre remarquable, lui, est l'objet de l'attention d'autres catégories d'acteurs : les promeneurs, les urbains, les journalistes, les historiens locaux. . . Tous cultivent, de façon curieuse, le culte de l'individu hors norme. Est-ce parce qu'ils sont finalement des symboles parfaits de la dimension géochronologique de la biodiversité (Arnould, Hotyat et Simon, 2000)? Ils s'imposent tout à la fois dans l'espace par leur port étrange, leur aspect spectaculaire, et ils se dressent comme des représentants du temps qui dure, en plus de toutes les autres charges symboliques qu'ils assument. Comment donc souhaiter la présence d'arbres remarquables, sans en devenir des idolâtreurs serviles et obtus? La voie est étroite. Elle suppose un dialogue entre des acteurs ayant une bonne culture naturaliste et des visiteurs porteurs de fortes doses de subjectivité et d'esthétisme, mais aussi la prise en compte des dimensions politiques, économiques et juridiques. Comme dans toute question environnementale, il faut parvenir à concilier des raisons et des visions contradictoires. Fontainebleau et Kampinos sont des lieux où se jouent les explications, les négociations et les possibles solutions de la gestion des arbres dans les forêts périurbaines.

\section{Remerciements}

Les auteurs remercient vivement Frédérique Lecomte et Marc Rumelhart pour leurs lectures attentives et leurs propositions constructives d'amélioration de leur manuscrit.

\section{Références}

Andrzejewski, R., 1994. Pomnikowe drzewa (Les arbres remarquables), Parki Narodowe, 2, 9-10 (en polonais).

Arkusz ewidencyjny pomnik przyrody Kampinoskiego Paruku Narodowego (fiches d'identification des arbres classés monuments de la nature du parc national de Kampinos) (en polonais, s. d.).

Arnould, P., 1986. Sous les mots, la forêt : recherches sur la toponymie et les paysages en forêt de Saint-Gobain et Coucy-Basse (02, Aisne), Lille, Hommes et Terres du Nord, 2-3, 221-226.

Arnould, P., 1997b. 15000 years of French and Polish woodland history: elements for comparison, in Ryszkowski, L., Wicherek, S. (Eds), Ecological management of countryside in Poland and France, Académie des sciences de Pologne, 4956.
Arnould, P., 1997c. Forêt et société. La forêt : un espace à la périphérie du monde social?, in Histoire de forêts. La forêt française du XIII au XX $X^{e}$ siècle, Paris, Centre historique des Archives nationales, Adam Biro, 117-133.

Arnould, P., 1997d. Arbres et forêts : images de pub, objet de mode?, in Corvol, A., Arnould, P., M. Hotyat (Eds), La forêt, perceptions et représentations, Groupe d'histoire des forêts françaises, Paris, L'Harmattan, 93-109.

Arnould, P., Hotyat, M., Simon, L., 1997a. Les Forêts d'Europe, Paris, Nathan.

Arnould, P. et al., 2000. Orientations régionales forestières. Île-deFrance, Paris, DRIAF Île-de-France.

Arnould, P., Hotyat, M., Simon, L., 2000. Biodiversity and forestry management. From biodiversity to geochronodiversity, in Agnoletti M., Anderson S., Methods and approaches in forest history, New York, CABI Publishing, 251-263.

Arnould, P., 2002. Fontainebleau, une forêt bâtie sur du sable, in Gadant, J. (Ed.), Atlas des forêts de France, Paris, J.-P. de Monza.

Arnould, M., Delfour, J., 2003. Fontainebleau, forêt capitale, Forêts magazine, rubrique "Forêt d'ici », 1, 24-41.

Association des amis de la forêt de Fontainebleau (AAFF), 1988. Arbres remarquables de la forêt de Fontainebleau.

Association des amis de la forêt de Fontainebleau (AAFF), 1998. Arbres remarquables de la forêt de Fontainebleau.

Atlas, 2002. La France et le monde, Paris, Nathan.

Ballion, R., 1975. La fréquentation des forêts, Revue forestière française, 27, 2, 155-170.

Balanger, L., 1989. À propos d'arbres remarquables ou « vous saurez tout sur le gros, le grand et le petit », Revue forestière française, 41, 3, 227-230.

Bourdu, R., Viard, M., 1988. Arbres souverains, Paris, Du May.

Brunet, R., Rey, V., 1996. Europes orientales, Russie, Asie centrale, Géographie universelle, Paris, Belin/Reclus.

Cans, R., 29 avril 1994. Jupiter décapité, Le Monde.

Colinet, C. Indicateur historique et descriptif de Fontainebleau, itinéraire de la forêt et des environs, 20e édition des Guides Denecourt (s.d.).

Corvol, A., 1987. L'Homme aux bois : histoire des relations de l'homme et de la forêt du XVII siècle au XX siècle, Paris, Fayard.

Denecourt, J.-C., 1854. Le Palais et la forêt de Fontainebleau. Guide historique et descriptif suivi d'un aperçu d'histoire naturelle de la forêt, $16^{\mathrm{e}}$ édition des indicateurs Denecourt.

Devèze, M., 1965. Histoire des forêts, Paris, PUF.

Dumas, R., 2002. Traité de l'arbre. Essai d'une philosophie occidentale, Arles, Actes Sud.

Dumé, G., Mansion, D., Rameau, J.-C., 1989. Flore forestière française. Guide écologique illustré, t. 1 : Plaines et collines, IDF.

Escurat, M., 1996. À propos des arbres les plus hauts de France, Revue forestière française, 48, 1, 87.

Falinski, J.-B., 1986. Vegetation dynamics in temperate lowland primaveral forests, Ecological Studies in Bialowieza forest, Dordrecht, Dr. W. Junk Publishers.

Falinski, J.-B., 1991. Le parc national de Bialowieza et le système intégral des espaces protégés en Pologne, Revue forestière française, $43, \mathrm{n}^{\mathrm{O}}$ spécial "Patrimoines naturels forestiers", 190-205.

Gougis, J.-M., 2000. Forêt domaniale de Fontainebleau. Révision de l'aménagement forestier 1996-2015, ONF.

Guittet, J., Laberche J.C., 1974. L'implantation naturelle du pin sylvestre sur pelouse xérophile en forêt de Fontainebleau. II. Démographie des graines et des plantules au voisinage des vieux arbres, Oecologia Plantarum, 9, 2, 111-130. 
Harrison, R., 1992. Forêts. Essais sur l'imaginaire occidental, Paris, Flammarion.

Hermeline, M., Rey, G., 1998. Lettre de la forêt de Fontainebleau. Abécédaire géographique, photographies d'A. Turlat, Textuel/ONF.

Heymanovski, K., 1966. Dzieje Pusczy Kampinoskiej do polowy XIX wieku (Histoire de la Puszcza de Kampinos jusqu'au milieu du XIX siècle), Sylwan, 90, 1-15 (en polonais).

Hotyat, M. 2003. Réserve biologique intégrale du Gros Fouteau en forêt de Fontainebleau : entre dynamiques contrôlées et dynamiques spontanées, Paris, CNRS-IHMC, Cahiers d'études, Forêts et réserves, 13, 35-43.

Kalaora, B., 1981a. Le Musée vert ou le tourisme en forêt, Paris, Anthropos.

Kalaora, B., 1981b. Naissance et développement d'un loisir urbain : la forêt de Fontainebleau, Études rurales, 83, 97-115.

Kalaora, B., 1983. Le génie d'un lieu, étude de deux exemples : la forêt d'Orléans et la forêt de Fontainebleau, Norois, 120, 583-590.

Kronika Kampinoskiego Parku Narodowego 1959-1962 (Chroniques du parc national de Kampinos de 1959 à 1962), t. 1, 1963 (en polonais).

Lecomte, F., 2001. Les arbres remarquables en forêt. Guide de gestion, Paris, ONF.

Lemée, G., 1989. Structure et dynamisme de la hêtraie des réserves biologiques de Fontainebleau : un cas complexe climatique de forêt feuillue monospécifique tempérée, Acta Oecologica, 10, 2, 155-174.

Lemée, G., 1990. Évolution du paysage de la forêt de Fontainebleau au cours des cinq derniers millénaires, Bulletin d'écologie, 21, 4, 119-127.

Lorgnier, A., 1992. Forêts, Marseille, AGEP.

Maresca, B., 2001. La Fréquentation des forêts publiques en Île-deFrance. Caractéristiques des sorties et flux de visites des franciliens. Étude réalisée dans le cadre de l'évaluation du contrat de plan État-région 1994-1999 de l'Île-de-France, Paris, Agence des espaces verts.

Misiak, J., 1994. Forests at the outskirts of towns: The Kampinos National Park, Naturopa, 75, 1-7.
Moigneu, T., Lecomte, F., 1997. Témoins des âges, les arbres remarquables des forêts publiques françaises: méthodologie d'inventaire et gestion durable, Paris, ONF, Les dossiers forestiers, contribution au congrès forestier mondial d'Antalya.

Moriconi-Ebrard, F., 1994. Géopolis : pour comparer les villes du monde, Paris, Economica, Anthropos.

Mosnier, J.-C., 1990. Encore des arbres remarquables, Revue forestière française, 42, 3, 375 .

ONF, 1993. Guide de prise en compte de la diversité biologique dans l'aménagement et la gestion forestière.

ONF, 1996. Note de service 96-T-131 du 12 juin sur le programme arbres remarquables.

ONF, 1997. Guide pour l'accueil du public dans les forêts relevant du régime forestier.

Parc national de Kampinos, 1996. Les Arbres remarquables du parc national de Kampinos.

Polton, J.C., 1995. Tourisme et nature au XIX ${ }^{e}$ siècle, Paris, CTHS.

Pourtet, J., 1979. Qu'est-ce qu'un arbre remarquable?, Bulletin de l'Association des parcs botaniques de France, 2, 10-11.

Rumelhart, M., 1999. À la conquête de l'infini ligneux, Les Carnets du paysage, 3, 74-101.

Silva, M.-A., 1997. La Signification de l'arbre pour la ville et les habitants de Genève. À l'exemple de certains arbres et traditions, Zurich, École polytechnique fédérale.

Simon, L., 2000. Les forêts de plaine en Europe : écologie, dynamisme et faits de répartition, L'Information géographique, 1, 37-57.

Simon, L., Mozgawa, J., Cieslak, C., 1997. La Pologne forestière entre permanences et mutations, Revue forestière française, $49,3,265-277$.

Touzet, G., 1994. La mort du chêne Jupiter, Le Monde, 14 mai.

Urbain J.-D., 2002. Paradis verts. Désirs de campagne et passions résidentielles, Paris, Payot.

Vallaeys, A., 2000. Fontainebleau. La forêt des passions, Paris, Stock.

Zestawienie zbiorcze pomnikow przyrody (Inventaire des arbres monumentaux du parc national de Kampinos), 1996. Kampinoski Park Narodowy (en polonais).

Reçu le 23 septembre 2003. Accepté le 11 mars 2004.

To access this journal online: www.edpsciences.org 